\section{Das Rheuma-Dispensaire}

Die sog. Dispensaires dienten in der ehemaligen DDR als Betreuungsstellen für Patienten mit rheumatischen $\mathrm{Er}$ krankungen. Hier erfolgte die ambulante Versorgung sowie die Behandlung. Auch für die weitere Unterstützung der Patienten waren die Dispensaires zuständig. Die Schließung der RheumaDispensaires war sowohl für die Betroffenen als auch die Ärzte in hohem Maße bedauerlich.

\section{Entwicklung des Gesundheits- wesens nach 1945}

Unmittelbar nach dem Ende des 2. Weltkrieges wurden in den 4 Besatzungszonen Deutschlands die bisherigen ministeriellen Strukturen abgeschafft. Besonders in der sowjetischen Besatzungszone erfolgte im Hinblick auf eine zukünftig aufzubauende sozialistische Staatsform eine massive Reform aller Verwaltungsbereiche. Dabei wurden auch die Strukturen des bisherigen Gesundheits- und Sozialsystem total verändert. Ein erster Schritt war die Verstaatlichung des Gesundheitswesens. In diesem Sinne erließ die sowjetische Militäradministration in Deutschland im Januar 1947 den Befehl 272 zur „Errichtung von Ambulanzen und Polikliniken zur Sicherstellung der ärztlichen Ver-

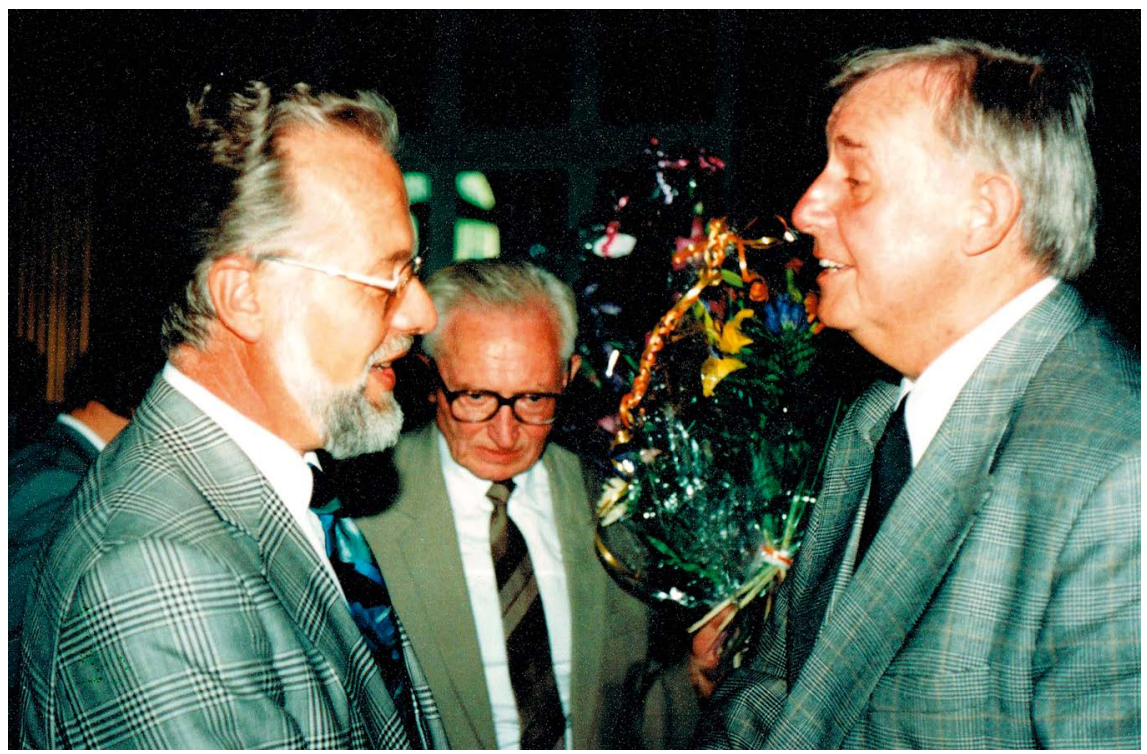

Prof. Dr. Hans-Egbert Schröder (links) und Prof. Dr. Gerhard Heidelmann (Mitte) bei der Verabschiedung von Prof. Dr. Heinrich Geidel aus dem Krankenhaus Dresden- Friedrichstadt 1994 (Quelle: Archiv, Klinikum Dresden). sorgung der deutschen Bevölkerung in der sowjetischen Besatzungszone“. Die Ausführung dieses Befehls übernahmen die deutsche Zentralverwaltung für das Gesundheitswesen und die örtlichen Gesundheitsämter. Nur so konnten der durch den Krieg bedingte Mangel an Ärzten und Praxiseinrichtungen leidlich kompensiert werden.

Darüber hinaus führte die Spezialisierung des Fachbereiches der Inneren Medizin in der ersten Hälfte des 20. Jahrhunderts zu einer enormen Erweiterung des Fachwissens. Eine Folge war Ende der 60er Jahre die Einführung einer sogenannten „Subspezialisierungen“ des Facharztes für Innere Medizin in einzelnen Fachbereichen, z. B. in Diabetologie, Kardiologie und auch Rheumatologie. Außerdem wurde ein „Facharztes für Allgemein-Medizin“ in der DDR eingeführt.

Die Zunahme an Wissen forderte eine erweiterte spezielle Fortbildung der Ärzte innerhalb des Grundfaches „Innere Medizin“. Für die Bedeutung einer „Subspezialisierungen" und der damit verbundenen speziellen Betreuung dieser Patientengruppen lieferte der Internist, Prof. Otto Rostowski, ein maßgebliches Beispiel. Anlässlich seines 90 . Geburtstages erinnerte er sich: „Als das Insulin zur Behandlung des Diabetes mellitus gefunden und die Behandlung in ganz neue Bahnen gelenkt worden war, eine Behand- lung, die damals den meisten Ärzten noch nicht geläufig sein konnte, habe ich 1924 das erste Ambulatorium für Diabetiker gegründet“. Es war das erste Diabetes-Ambulatorium in Europa (2). Ähnliche Ambulanzen wurden bereits damals für bestimmte Blutkrankheiten, für Hypertonie und verschiedene andere Leiden, allerdings vorwiegend an Universitäts-Kliniken, gegründet.

\section{Etablierung der Rheuma-Dispensaires}

Dispensaires waren keine politische Erfindung der DDR. Bereits im 19. Jahrhundert gab es in den französischen Hospitälern solche Einrichtungen zur Betreuung chronisch Kranker. Daher sicher die übernommene französische Bezeichnung. Im Rahmen des Rheumabekämpfungs-Programms der DDR, das bereits Anfang der 50er Jahre im Hinblick auf die Beherrschung des „Rheumatischen Fiebers und seiner Folgeerscheinungen“ eingeführt worden war, erfolgte daher die Berufung von „Fachberatern für Rheumatologie“ in allen 15 Bezirken der DDR. In der Folge etablierten sich Beratungsstellen für Rheumatologie (Dispensaires) auch in fast allen Kreisen der DDR. Die ärztlichen Stellen waren einem Amts(Kreis)-Arzt unterstellt und mit einem spezialisierten Facharzt für Innere Medizin vorwiegend neben-, aber auch hauptamtlich, zu besetzen. Als sehr wichtig wurde eine enge fachliche Anbindung der Physio- und Ergotherapie sowie der Sozialfürsorge an diese Stellen angesehen. Neben einem Leiter, im allgemeinen einem Internisten mit der Subspezialisierung „Rheumatologie“ waren in den jeweiligen Beratungsstellen auch rheumachirurgisch arbeitende Fachärzte für Orthopädie und Fachärzte für Pädiatrie konsiliarisch tätig.

Eine Forderung war, für besondere diagnostische und therapeutische Maßnahmen 4-5\% der Krankenhausbetten für die entsprechenden Patienten bereitzuhalten. Die Überweisung der Patienten in das Rheumadispensaire erfolgte durch die in der peripheren Praxis tätigen Kollegen mit einer entsprechenden Fragestellung zur konsiliarischen Mitbehandlung. Die allgemeine medizinische Betreuung blieb aber unverändert beim Hausarzt. 
Die Aufgaben der Rheumadispensaires wurden nach Marcusson (3) bereits 1945 klar festgelegt:

- allgemeine Krankheitsverhütung, was besonders für das Rheumatische Fieber galt

- Früherkennung und Differenzierung der verschiedenen Arthritiden

- Betreuung der bereits Erkrankten

- nachgehende Fürsorge zur Vermeidung von Behinderungen und Pflegebedürftigkeit.

In der DDR wurden so in allen damals bestehenden 15 Bezirken Beratungsstellen mit beratenden Rheumatologen als territoriale Zentren der Rheumabekämpfung aufgebaut. Erweitert wurde dieses Netzwerk durch Rheumatologische Dispensaires in den Kreisen. So bestanden im Jahre1987 in den verschiedenen Kreisen 248 lokale Zentren.

\section{Wissenschaftliche Zusammenarbeit}

Für die wissenschaftliche Anleitung und Fortbildung waren die Akademie für Ärztliche Fortbildung der DDR und die Gesellschaft für Rheumatologie in der DDR verantwortlich. Sie erfolgte durch Fortbildungsabende, Seminare und Kongresse. Besonders intensiv und nachhaltig war eine Form des „Ganztagspraktikums“, bei dem die Teilnehmer in der Klinik 1 Woche lang täglich vormittags auf der Rheuma-Station die Visite und nachmittags die klinischen Untersuchungen, Besprechung und Diskussionen der Röntgen- und Laborbefunde miterleben und gestalten konnten.

Mit der Übernahme der klinischen Betreuung der Rheuma-Patienten und den Aufgaben der Rheumadispensaires des Bezirks Dresden wurde die I. Medizinische Klinik Dresden-Friedrichstadt 1973 beauftragt. Die klinische Differentialdiagnostik und Therapie sollte dort auf einer spezialisierten Station erfolgen. Günstig war, dass das Labor der genannten Klinik seit 1961 durch Prof. Beickert intensiv immunologisch profiliert wurde. So war eine Differenzierung der einzelnen Formen der rheumatologischen Erkrankungen relativ schnell möglich.
Andererseits war durch die Struktur des Klinikums eine enge konsiliarische Zusammenarbeit mit den verschiedenen Fachrichtungen der Physiotherapie, der Radiologie, der Orthopädie und Chirurgie, der Dermatologie sowie der Pathologie bei unklaren Fragestellungen in Klinik und Therapie gegeben.

\section{Ambulante Betreuung}

Die ambulante Betreuung im Dispensaire erfolgte mehrmals, im Allgemeinen 2-mal pro Woche in einer poliklinischen Einrichtung in der Dresdener Südvorstadt durch einen Internisten mit der Subspezialisierung Rheumatologie. In der Ambulanz erfolgte eine gründliche, zeitlich nicht begrenzte Erhebung der Anamnese, in der auch berufliche und soziale Fakten angesprochen und beachtet wurden. An dieses erste Gespräch, in dem man schon einen guten Kontakt zu dem Patienten aufbauen konnte, folgte eine sorgfältige und eingehende klinische Untersuchung. Daneben stand natürlich eine exakte Beurteilung der Funktionen des Bewegungsapparates im Vordergrund des Interesses. Zur Dokumentation der Befunde verwendeten wir im Laufe der Zeit durchaus wechselnde Indizes. Anfangs war es der LANSBURY-Index, später der Funktionstest nach KEITEL und der Disease Activity Score, für die Kranken mit einer Spondylitis ankylosans die Selbstauskunfts-Bögen BASDAI und BASFI, die später quantitative Vergleiche und Kontrollen ermöglichten.

Im Verlauf der ersten Konsultation wurde auch eine Blutentnahme zur Einschätzung der Entzündungsaktivität (Blutsenkung, Blutbild, Elektrophorese, ) und zum ev. Nachweis von spezifischen Antikörpern (Rheumafaktoren, LE-Zellen, u.ä.) vorgenommen, um den Patienten, die oft längere Wege zur Ambulanz zurückzulegen hatten, körperliche Belastungen durch eine erneute Vorstellung zu ersparen.

Schon bei der Vereinbarung eines Untersuchungstermines wurde um die Vorlage vorhandener Röntgenaufnahmen gebeten. Wichtiger als Ergebnisse der bildgebenden und Labor-Untersuchungen waren aber neben den aktuellen klinischen Befunden und Gelenkfunktionen auch das psycho- physische Verhalten des Kranken. Wie wurden die Beschwerden geschildert? Wie war seine Stimmung, gelöst oder eher depressiv? Wie war der Gang? Konnte er sich flüssig entkleiden? Äußerte er dabei Schmerzsymptome? Welche Behinderungen bestanden?

\section{Pharmakotherapie}

Die Durchführung der Penicillin-LangzeitProphylaxe beim Rheumatischen Fieber war eine der ersten angeordneten, größeren Maßnahmen im Rheumadispensaire. Da die regelmäßige Einnahme von Tabletten nicht bei allen Patienten gesichert war, erfolgten die Injektionen des Medikamentes im regelmäßigen Rhythmus während der Sprechstunden im Dispensaire oder wurden durch den Hausarzt vorgenommen.

Die Behandlung der Schmerzen, die auch bei den chronischen Gelenkerkrankungen in den meisten Fällen recht akut sein können, erfolgte in diesen Jahren mit Acetylsalizylsäure, Paracetamol und zunehmend mit Phenylbutazon und anderen nichtsteroidalen Antirheumatika. Dazu kamen damals relativ großzügig Glukokortikoide, auch als Dauertherapie in einer niedrigen Dosierung zum Einsatz, deren Nebenwirkungen wir kannten, aber als beherrschbar ansahen. Zusätzlich begannen wir bei einer entsprechenden Indikation mit einer „Basistherapie“ mit dem Chlorochin oder mit Goldinjektionen.

Da die Langzeitergebnisse dieser Therapie in zahlreichen Fällen nicht überzeugend waren, begannen wir schon Ende der 1960er-Jahre bei hoch akuten, destruierenden Verläufen mit einer immunsuppressiven Therapie mit Methotrexat oder Cyclophosphamid. Die klinischen Ergebnisse waren beachtenswert und ermutigten uns, diese Medikamentengruppe schärfer ins Visier zu nehmen.

Da die Therapie mit Gold-Präparaten und Glukokortikoiden, besonders aber mit den immunsupressiv wirkenden Medikamenten ein gewisses Maß an Spezialwissen voraussetzte, empfahlen wir in den meisten Fällen die medikamentöse Einstellung unter stationären Bedingungen, um die Verträglichkeit und eventuelle Nebenwirkungen rechtzeitig erkennen und entsprechend handeln 
zu können. Nach dem Erreichen einer stabilen Phase im Krankheitsverlauf erfolgte dann die weitere spezielle Betreuung und Laborkontrollen im Rheumadispensaire.

Verschiedene Pharmaka aus der Gruppe der Antirheumatika mussten importiert werden und standen nur beschränkt zur Verfügung. Deshalb war durch die Bezirksverwaltung zeitweise eine Verordnung nur durch den Arzt im Rheumadispensaire angeordnet worden. Dadurch wurde gesichert, dass nur Bedürftige eine entsprechende Therapie erhielten

Sollte sich der Zustand des Patienten unter der eingeschlagenen Therapie verschlechtern, die Schmerzen zunehmen oder eine Veränderung des Allgemeinbefindens auftreten, konnte er sich jederzeit, auch ohne Anmeldung bei „seinem Rheumatologen“ vorstellen. So konnte kurzfristig reagiert werden.

\section{Weitere Therapien}

Neben der Pharmakotherapie war die 2. wichtige Säule der Behandlung die Physiotherapie. Durch die Schaffung einer eigenständigen Fachrichtung und eines „Facharztes für Physiotherapie“ wurde eine wesentliche Lücke im Behandlungsplan geschlossen. Mit der konsiliarischen Bindung von physiotherapeutischen Fachkräften an die klinischen Abteilungen und Rheumadispensaires war eine zielgerichtete und erfolgversprechende Behandlung möglich. Spezielle Bewegungsübungen und elektro- physiologische Maßnahmen mit unterschiedlichen Stromformen wurden in regelmäßigen Konsilen in den Kliniken und Ambulanzen in die Behandlungspläne eingebaut. Nach Beobachtungen über einen längeren Zeitraum haben wir die gewonnenen Erkenntnisse bei Kongressen und anderen Fortbildungsveranstaltungen zur allgemeinen Anwendung vorgestellt.

Auch durch gezielte Einsätze der Ergotherapie konnten nicht nur physisch, sondern auch psychisch Fortschritte im Verlauf zu einer Remission erzielt werden. Welche Bedeutung die Psyche für eine erfolgversprechende Therapie haben kann, wurde uns in einer außergewöhnlichen Situation im klinischen Alltag deutlich. Die Stimmung im Frauensaal mit 11 Rheumatikerinnen war in einem trüben November miserabel. Die Pa- tientinnen klagten ständig über multiple, klinisch kaum verifizierbare Schmerzen und Beschwerden. Eines Tages nahmen wir eine jüngere, lebensmutige Frau, Leiterin eines Jugendhauses, wegen einer aktiven rheumatoiden Arthritis auf. Sie erlebte diesen deprimierenden Zustand einen halben Tag und besorgte sich dann einige Utensilien aus ihrer Arbeitsstelle. Der nächste Morgen begann mit einer Überraschung. Wir Ärzte wurden „offiziell ins Zimmer geführt“ und mussten zunächst eine kleine „Galerie“ auf dem großen Zimmertisch betrachten, welche die Frauen aufgebaut hatten: Da standen kleine Kerzenleuchter, Nussknacker, Pappschächtelchen und andere kleinere, einfache Sachen, nett anzusehen, weil offensichtlich „mit Liebe“ gemacht. Die Patientinnen hatten erlebt, dass sie etwas schaffen und sich darüber freuen können, trotz schmerzhafter Behinderungen. Sie hatten ein neues Selbstwertgefühl bekommen, das ihnen in der Annahme ihres Leidens geholfen hatte. Zukünftig spielte die Ergotherapie eine wichtige Rolle im Therapieplan. Wir sagten bewusst Ergotherapie statt Arbeitstherapie, weil unsere männlichen Patienten meinten, bei einer Arbeitsunfähigkeits-Bescheinigung dürfe man auch keine „Arbeitstherapie“ machen! Sie ließen sich aber rasch bekehren und fertigten, als sie den Wert der Maßnahme erkannten sehr schöne handwerkliche Gegenstände.

Wir erkannten auch, dass die Kranken mehr über ihre Krankheit wissen wollten. Um diese Wissenslücken zu beseitigen, veranstalteten wir einmal wöchentlich RheumaSeminare, in denen unsere Kollegen über die medikamentöse, über die Physio- und Ergotherapie sowie über psychische Symptome bei rheumatischen Erkrankungen referierten und Fragen beantworteten.

\section{Unterstützung der Patienten}

Selbsthilfe-Gruppen oder „ähnliche Vereine“ zu bilden, war aber in der DDR nicht erlaubt. Auch hier übernahm das Dispensaire eine helfende Funktion. Wir haben für interessierte Patienten „Gesprächskreise“ im Rahmen des Rheumadispensaires gebildet, wo über alle Probleme gesprochen werden konnte, die wir ärztlicherseits kaum oder gar nicht beantworten konnten. Welche Wege im Dschungel der Verordnungen in welcher Reihenfolge zu gehen waren, wussten wir Ärzte nicht so genau. Eine Hilfe konnten hier die „Gesprächskreise“ durch die Mund zu MundPropaganda bieten, denn in vielen Fällen waren Patienten aus eigener Erfahrung über das entsprechende Prozedere vorzüglich informiert. Unsere Patientinnen nahmen das dankend an.

Ein anderes Problem bestand darin, dass es in der DDR üblich war, Trainingszeiten in Schwimmhallen nur an Leistungs-Sportvereine zu vergeben. Unsere Bechterew-Patienten wurden mit ihrer Bitte um Zuteilung einer Übungszeit für ihre, als ganz wesentliche Therapie anzusehende Wassergymnastik nicht erhört. Eine Selbsthilfe-Gruppe durften wir ja nicht gründen. Ergo musste man eine andere „Lücke“ finden. Die „Bechtis" traten einem Sportverein in Dresden bei und gründeten eine Sektion „Versehrten-Sport“. Das klappte! Der Verein freute sich über neue Mitglieder und ermunterte die Betroffenen, fleißig zu trainieren, um demnächst an den Paralympics teilzunehmen. Wir hatten unsere Übungszeit und haben nur geschmunzelt.

Im Laufe der Zeit entwickelte sich in zunehmendem Maße eine Zusammenarbeit mit den Orthopäden. In den frühen Jahren waren Konsile zur Indikation einer Synovektomie als aktiven Eingriff in den entzündlichen Gelenkprozess vordergründig. Später wurde besonders bei den degenerativen Formen der Gelenkerkrankungen über Operationen von Fehlstellungen verschiedener Gelenke bis zum Gelenkersatz gemeinsam beraten. Profitiert haben alle: die Patienten, die eine qualifizierte Beratung, ja manchmal sogar einen OP-Termin erhielten und die Ärzte, für die das Konsil eine effiziente Form der Fortbildung war.

Wesentliches Probleme, die speziell im Rheumadispensaire immer wieder auftraten, waren Fragen zur Arbeits- oder Berufsfähigkeit, zu Indikationen verschiedenartiger rehabilitativer Maßnahmen, z. B. zu Kuren oder zum Grad der Behinderung, die viele rechtliche Voraussetzungen als Grundlage hatten. Deshalb war die Anstellung einer Fürsorgerin (was m.E. besser als „Sozialarbeiterin“ klingt) sehr vorteilhaft. Die gesetzlichen Voraussetzungen für die Gewährung der notwendigen bzw. erwünschten Maßnahme und deren Beantragung waren den in der Praxis tätigen Kollegen nicht voll- 
ständig geläufig und mit einer bürokratischen Zusatzleistung verbunden. Die Übernahme dieser Aufgaben durch unsere Fürsorgerinnen löste die Probleme.

Die Betreuung durch das Dispensaire erfolgte kontinuierlich so lange wie nötig in Abständen von 1 bis 2 Wochen bis zu einem halben Jahr, je nach der Aktivität der Erkrankung.

Die oft über viele Jahre erhobenen Daten bildeten eine Grundlage für wissenschaftliche Aussagen zu den Langzeitverläufen, den Ergebnissen von Behandlungsmaßnahmen und die allgemeine und soziale Bedeutung der Erkrankungen des rheumatischen Formenkreises.

Jährlich musste ein Bericht über die geleistete Arbeit und die Ergebnisse, Zahl der Kranken in den einzelnen Entitäten, Ergebnisse der Therapien, Auftreten von unerwünschten Nebenwirkungen u.a. erstellt werden.
FAZIT

Durch die Arbeit im Rheuma-Dispensaire kannte ich die Verläufe einschließlich aller Probleme um das Krankheitsgeschehen bei meinen Patienten über lange Zeit, bei einzelnen Kranken über mehr als 30 Jahre. Die Schließung der RheumaDispensaires mit dem Beitritt der DDR zur Bundesrepublik war sowohl für die Betroffenen wie die Ärzte in einem hohen Maße bedauerlich.

\section{Interessenkonflikt}

Der Autor gibt an, dass kein Interessenkonflikt besteht.

Autor

Heinrich Geidel
Korrespondenzadresse

Prof. Dr. Heinrich Geidel

Friedrichstraße 52

01067 Dresden

Deutschland

\section{Literatur}

Weiterführende Literatur beim Verfasser.

Bibliografie

Akt Rheumatol 2020; 45: 513-517

DOI 10.1055/a-1252-3300

ISSN 0341-051X

C 2020. Thieme. All rights reserved.

Georg Thieme Verlag KG, Rüdigerstraße 14, 70469 Stuttgart, Germany 